\title{
Consideraciones y estrategias para la implementación de un sistema de gestión de la calidad ISO 9001 en el marco del Licenciamiento y la Acreditación de la Educación Superior Universitaria en el Perú
}

\section{Considerations and strategies for the implementation of a quality management system ISO 9001, in the framework of licensing and accreditation of university higher education in Perú}

\author{
Pedro Angulo Herrera ${ }^{1, *}$, Pedro J. Angulo Alvarado ${ }^{2}$, Elizabeth Coronel Capacyachi ${ }^{3}$, Juan A. Espinoza Blanco ${ }^{1}$ \\ ${ }^{1}$ Universidad Nacional Mayor de San Marcos, ${ }^{2}$ Kaizen Certification ${ }^{3}$ Universidad Continental
}

\section{RESUMEN}

La nueva Ley Universitaria ha marcado un antes y un después en el contexto educativo peruano con prospectiva hacia un mercado más competitivo. El plan de gestión de la calidad del Licenciamiento y, el sistema de gestión de la calidad (SGC) de la acreditación propician el cambio funcional de la universidad hacia una gestión sistémica basada en procesos. Sin embargo, antes de la implementación del SGC es necesario algunas consideraciones como żqué es calidad y para qué?, żcuál es la finalidad de la educación en nuestro medio?, ¿̇qué es educación con calidad o calidad educativa?, ¿̇qué es un plan de gestión de la calidad? ¿̇qué es gestión de la calidad?,¿̇qué es un sistema de gestión de la calidad? ¿̇qué es el aseguramiento de la calidad? La universidad tiene su propia normatividad, su propia política de aseguramiento de la calidad y sus propios comportamientos que la hace diferente a las demás organizaciones; por eso, en este nuevo contexto, proponemos algunas consideraciones y estrategias para la implementación, de acuerdo a la Norma ISO 9001 de un SGC en las universidades y sus programas de estudios. Esta implementación no debe ser por moda, obligación, status, o marketing; debe ser para demostrar con evidencia que las actividades de la organización educativa se hacen de manera congruente y con mejora continua para lograr la excelencia en la gestión de la profesionalización del talento humano para la empleabilidad, la generación de conocimiento con calidad y el desarrollo de la innovación en nuestro país.

Palabras claves: Sistema de Gestión de Calidad, licenciamiento, acreditación, ISO 9001 en universidades.

\begin{abstract}
The new University Law has marked a before and after in the peruvian educational context with prospective towards a more competitive market. The quality management plan of the Licensing and the quality management system (QMS) of the accreditation facilitate the functional change of the university towards systemic management based on processes. However, before the implementation of the QMS is necessary some considerations such us What is quality and what for?, What is the purpose of education in our environment?, What is education with quality or educational quality?, What is a quality management plan?, What is quality management?, ¿What is a quality management system? What is quality assurance? The university has its own regulations, its own policy of quality assurance and its own behaviors that makes it different from other organizations; therefore, in this new context, we propose some considerations and strategies for implementation, according to the ISO 9001 standard of a QMS in universities and their study programs. This implementation should not be for fashion, obligation, status, or marketing; it must be to show with evidence that the activities of the educational organization are done in a consistent manner and with continuous improvement to achieve excellence in the management of the professionalization of human talent for employability, the generation of knowledge with quality and the development of the innovation in our country.
\end{abstract}

Keywords: Quality management system, licensing, accreditation, ISO 9001 in universities.

Historial del artículo:

Recibido, 04 de marzo 2016; aceptado, 10 de mayo de 2016; disponible en línea, 15 de julio de 2016

* Docente de la Universidad Nacional Mayor de San Marcos.

Correo: pedro_qf@hotmail.com 


\section{INTRODUCCIÓN}

La organización funcional del sistema universitario peruano pasa por un antes y un después. La línea divisoria es la Ley Universitaria 30220 que establece al Ministerio de Educación (MINEDU) como el ente rector de la Política de Aseguramiento de la Calidad de la Educación Superior Universitaria (Política de Aseguramiento) y se crea la Superintendencia Nacional de Educación Superior Universitaria (SUNEDU) para aprobar o denegar las solicitudes de licenciamiento de universidades, filiales, facultades, escuelas y programas de estudios conducentes a un grado académico. El Sistema Nacional de Evaluación y Certificación de la Calidad Educativa (SINEACE) ha publicado un (nuevo) Modelo de Acreditación para Programas de Estudios de Educación Superior Universitaria (Modelo de Acreditación).

Entonces, el licenciamiento y la acreditación se convierten en instrumentos esenciales para introducir en la universidad una gestión orientada a la calidad y su mejora continua.

Pero también, esta nueva regulación por parte del Estado cambia el marco normativo para que las universidades públicas y privadas, nacionales y extranjeras, así como las filiales sigan ofreciendo el servicio educativo superior universitario. Ahora, el funcionamiento de una universidad y sus programas deben ser licenciados (habilitados) y, su calidad debe ser acreditada para que los usuarios del servicio tengan el beneficio de una educación con calidad y el país cuente con el capital humano necesario para su desarrollo productivo y posicionamiento internacional.

\section{Desafíos del nuevo entorno educativo}

Este nuevo escenario hace más competitivo al mercado educativo y su impacto puede llegar hasta la decisión de continuar operando o no. Quienes deseen continuar tienen que asumir los costos de inversión en infraestructura, mejoramiento de las condiciones para la investigación, ampliación de servicios complementarios para los estudiantes, etc., para adecuarse a las condiciones básicas de calidad (CBC) obligatorias para el licenciamiento y avanzar hacia la acreditación en el marco del aseguramiento de la calidad de la educación superior universitaria.

Estos cambios, definitivamente, tienen un impacto directo en la estructura organizacional, funcional, estratégica, táctica, financiera, curricular, etc. de las instituciones universitarias, para lo cual muchas no están preparadas para este desafío. Tendrán que experimentar diversas restructuraciones para poder seguir funcionando en un mercado con más de un millón trescientos mil estudiantes que se apertura a la inversión extranjera y cuya meta es la excelencia académica.
A diferencia de las organizaciones educativas, las organizaciones empresariales, tienen la experiencia suficiente y saben como actuar frente a estos cambios en el mercado. Por ejemplo, la XI Encuesta de gerentes generales (2015) mostró que, frente a la desaceleración de la economía peruana, la mitad de gerentes tiene por prioridad principal mejorar la eficiencia de los procesos. Lino Abram, socio de McKinsey Perú, manifestó (...). Si no se cambia la forma como se opera, no se lograrán las eficiencias a largo plazo (Semana Económica, 2014).

Mejorar la eficiencia de los procesos y mejorar la productividad no es nada nuevo en el mundo empresarial; sin embargo, nuestras organizaciones educativas no tienen esa experiencia y su gestión funcional con sesgos de la era industrial puede ser un impedimento crítico para este cambio.

\section{Reorganización y cambio en las organizaciones educativas como una prioridad}

Si hay un nuevo entorno, entonces el cambio es una prioridad. Por eso, el Modelo de Licenciamiento indica el mayor reto que enfrenta la sociedad peruana en materia de educación superior universitaria es el de reorganizar el sistema universitario y promover uno basado en calidad. Además, ...este complejo proceso implica un cambio institucional que requiere nuevos procedimientos y mecanismos, a fin de conseguir nuevos resultados (SUNEDU, 2015). Entonces, la reorganización y el cambio en las organizaciones educativas universitarias es condición sine qua non para adaptarse al nuevo escenario.

La situación requiere de un liderazgo efectivo para la gestión del cambio y cumplir con la misión y propósito de formar profesionales de calidad; así como también, se debe poner atención en la importancia que tiene la gestión de la cultura organizacional en el alineamiento del recurso humano para la gestión estratégica del programa de estudios.

Estos cambios alcanzan a muchos aspectos de la vida académica; en primer lugar, la institución educativa debe lograr la máxima satisfacción del cliente (calidad del servicio al cliente) porque el estudiante es el centro de atención de la reforma universitaria. El estudiante es sujeto de derecho a una educación superior integral y de calidad que le permita insertarse con dignidad en el mundo del trabajo, ser productivo, competitivo y valorado por el mercado laboral. En segundo lugar, debe adecuar e implementar toda la infraestructura necesaria para lograr este primer acometido, debe hacer uso de todas las herramientas de gestión para que sea valorada por la sociedad (MINEDU, 2015) a través de un proceso de transparencia. 
Como dijo Charles Darwin "no es la más fuerte de las especies la que sobrevive y tampoco la más inteligente. Sobrevive aquella que más se adapta al cambio". Las organizaciones que son capaces de adaptarse a las nuevas circunstancias, a las nuevas necesidades de sus clientes y sobre todo que sepan anticiparse y rediseñar su organización para crear acciones estratégicas adaptativas se convertirán en referentes en su sector, y en cualquier caso tendrá un mayor porcentaje de supervivencia (Evoluciona, 2015).

En el desafío de este nuevo escenario, SUNEDU otorgó la primera Licencia de Funcionamiento Institucional a la Universidad de Ingeniería y Tecnología (UTEC) que se presentó voluntariamente a la evaluación antes de la fecha prevista y obtuvo su licencia antes del límite de los plazos fijados para este proceso (Universidad de Ingeniería y Tecnología, 2016). Esta universidad para obtener su licenciamiento ha cumplido con todas las condiciones que se requieren para el licenciamiento, entre ellos el Plan de Gestión de la Calidad / Plan de mejora continua.

\section{Hacia una organización basada en procesos y gestión del cambio}

La educación es un derecho humano fundamental y un bien público irrenunciable $y$, debe ser de calidad; por lo tanto, se debe gestionar su calidad. Entonces, ¿̇qué debe caracterizar a la organización que gestiona su calidad?. En la Política de Modernización de la Gestión Pública al 2021 se indica que la gestión pública moderna es una gestión orientada a resultados al servicio del ciudadano; una gestión al servicio del ciudadano necesariamente deberá cambiar el tradicional modelo de organización funcional y migrar hacia una organización por procesos, que asegure que los bienes y servicios públicos de su responsabilidad generen resultados e impactos positivos para el ciudadano, dados los recursos disponibles (Presidencia del Consejo de Ministros, 2013).

Los procesos cruzan la jerarquía funcional de la organización, propician el trabajo en equipo y van creando una manera distinta de hacer las cosas con orientación a los resultados de acuerdo a la exigencia del mercado. Siendo los procesos repetitivos se presenta una oportunidad de mejora continua con innovación productiva en beneficio del cliente y adaptación al entorno (Agudelo, 2012).

El anterior modelo de CONEAU (SINEACE) fue diseñado aplicando el enfoque sistémico, a través del cual se representan todas las interacciones de los procesos que tienen lugar en la unidad académica y que le permiten alinearse al cumplimiento de los compromisos adquiridos por la institución con la sociedad en cuanto al conocimiento creado, los profesionales formados y los servicios entregados a la comunidad (CONEAU, 2008). Un indicador de calidad de este modelo era el sistema de gestión de calidad de la Unidad Académica es eficaz, y un estandar fue la Unidad Académica tiene un sistema de gestión de calidad implementado.

Este modelo marcó la pauta para un cambio de una organización educativa funcional (tradicional) a una organización sistémica articulada por procesos, poniendo énfasis en el proceso de formación profesional. Este modelo también tuvo en cuenta la gestión de la cultura organizacional, muy importante para la gestión del cambio en las personas para la adaptación de la organización a los nuevos desafíos de la globalización.

En el tiempo de vigencia de este modelo ha quedado demostrado que lo más difícil en nuestro medio es producir el cambio, żqué nos garantiza que ahora no se produzca lo mismo sino se hace la gestión del cambio?

\section{Se requiere de gestores y líderes del cambio}

La Política de Aseguramiento toma en cuenta a la gestión universitaria para enfrentar a los desafíos actuales de la educación superior universitaria, la universidad requiere contar con gerentes universitarios competentes para gestionar institucionalmente la formación integral que comprende la profesionalización del talento humano y la producción de conocimiento de alta calidad, además de los medios necesarios que ambos procesos requieren (MINEDU, 2015).

En el Modelo de Acreditación, en lo que se refiere a los recursos humanos para la gestión del programa de estudios, el estándar indica que el grupo directivo o alta dirección del programa de estudios está formado por profesionales calificados que gestionan su desarrollo y fortalecimiento. Siendo el criterio a evaluar la gestión de los recursos humanos de acuerdo con los perfiles de puestos y funciones establecidas. Además, para el aseguramiento de la calidad, establece que el programa de estudios implementa un sistema de gestión de la calidad y se compromete con la mejora continua y fija en la matriz de estándares el sistema de gestión de la calidad y los planes de mejora (SINEACE, 2016).

Al considerar que la organización educativa debe ser gestionada por gerentes universitarios competentes y que la alta dirección esté formado por profesionales calificados el Estado se asegura que se den los cambios al interior de la organización y se desarrolle una cultura de la calidad. Además, la implementación y certificación de un sistema de gestión de la calidad, en base a la norma ISO 9001, garantiza la eficiencia de los procesos y la mejora continua. 
La norma ISO 9001 en su versión 2015 amplia el alcance del SGC para proporcionar un enfoque integrado de la gestión organizacional a partir del contexto de la organización y las partes interesadas (grupos de interés).

\section{Consideraciones previas para la implementación de un SGC ISO 9001}

La implementación de un sistema de gestión de la calidad, de acuerdo a la ISO 9001, es imperativo en la universidad peruana para facilitar su proceso de adaptación al nuevo escenario marcado por el logro de una educación con calidad para la generación de conocimiento con calidad, el desarrollo de la innovación y la profesionalización del talento humano para la empleabilidad.

Por eso, antes de exponer algunas consideraciones para la creación y la implementación de un SGC orientado hacia la excelencia de todo el sistema universitario peruano es necesario ubicar el tema en el contexto académico actual que está marcado por las posiciones de las instituciones que participan en los pilares del sistema de aseguramiento de la calidad. Nos referimos a las publicaciones, que se han realizado, como resultado de las propuestas de equipos de calidad y de expertos en el tema, de MINEDU, SUNEDU, SINEACE y otros.

\section{¿Qué es calidad y para qué?}

Para el comité de calidad del SINEACE, la calidad es un concepto socialmente producido. La calidad es un significante, no un significado, que puede adquirir múltiples significados que dependen de la perspectiva social, de los sujetos y del contexto. Es históricamente producido, por lo que no puede definirse en términos esenciales, ni absolutos, pero tampoco es un concepto neutro. Por eso, calidad es una categoría que se mueve desde la subjetividad al ajuste científico, entre cultura y tekné, entre poiesis y repetición; entre crear y producir. Como poiesis, la educación para cumplir la finalidad de su razón de ser deberá ser de calidad. La educación poiética con calidad es acción transformadora y productora - reproductora de calidad (SINEACE, 2015).

Nos parece que esta definición se hace desde la perspectiva del paradigma de la complejidad (Morin, 2002) y marcan distancia con lo que llaman "calidad en la cultura productivista" que caracteriza a la cultura industrial.

Sin embargo, para la Política de Aseguramiento, la calidad es la búsqueda de la excelencia a través del esfuerzo continuo que se hace visible en la eficiencia de los procesos, en la eficacia de los resultados y en la congruencia y relevancia de estos procesos y resultados con las demandas y expectativas sociales. Además, uno de los principios de la Política de Aseguramiento es la calidad y excelencia académica, que define a la calidad como el grado de ajuste entre las acciones que una universidad, programa académico o carrera lleva a cabo para implementar las orientaciones contenidas en su misión y propósito institucional y los resultados que de estas acciones consigue (MINEDU, 2015).

Según el Modelo de Licenciamiento este propósito tiene dos dimensiones. La consistencia externa es el ajuste a las exigencias del medio externo (mercado laboral, la comunidad académica y el entorno socioeconómico de cada institución de educación superior) que asegura la pertinencia del propósito y permite comparar instituciones y programas. La consistencia interna es la traducción de las exigencias mencionadas en función de las prioridades y principios de la institución de educación superior, que le otorga mayor capacidad de proponer, innovar y ampliar la oferta en el sistema de educación superior (SUNEDU, 2015).

Podemos, inicialmente, concluir que un sistema de gestión de la calidad debe asegurar que se cumpla la misión y propósito institucional y, sus resultados.

\section{Dos enfoques de la calidad, żdiferentes o complementarios?}

Parecería que, el enfoque de la Política de Aseguramiento y del SINEACE son diferentes, pero nos parece complementarios. Para la Política de Aseguramiento, calidad es la búsqueda de la excelencia mediante la mejora continua de la eficiencia de los procesos, en la eficacia de los resultados y su pertinencia con las demandas sociales; mientras que para el SINEACE la calidad se centra en la persona, qué tipo de personas queremos formar y qué tipo de sociedad aspiramos tener. La calidad en educación se evidencia en una formación integral y en su contribución al desarrollo; demostrar que algo es de calidad es aludir a su valor (SINEACE, 2016).

La posición del SINEACE va en línea con la definición que hace la Ley General de Educación sobre calidad de la educación como el nivel óptimo de formación que deben alcanzar las personas para enfrentar los retos del desarrollo humano, ejercer su ciudadanía y continuar aprendiendo toda la vida (MINEDU, 2003).

Para la Política de Aseguramiento, la calidad se sustenta en la mejora continua de los procesos lo cual se hace mediante la implementación de un sistema de gestión de la calidad y el ciclo de la mejora continua de Deming (Agudelo, 2012). Por eso, la Política de Aseguramiento considera como una acción estratégica para la construcción del Sistema de Aseguramiento de la Calidad de la Educación Superior Universitaria, 
El Ministerio de Educación, en coordinación con la universidad, promueve la creación e implementación de un sistema de gestión de la calidad en todo el sistema universitario (MINEDU, 2015).

Este enfoque es parecido a lo que sucede en las empresas (Benzaquén, 2013). Sin embargo, para el Comité de Calidad del SINEACE "la escuela no es una empresa" (SINEACE, 2015).

Es necesario considerar que tanto en una empresa como en una institución educativa hay personas que realizan actividades enmarcadas en procesos. Estos procesos pueden ser mejorados y optimizados en un sistema de calidad. Este sistema de calidad puede ser evaluado (interna y externamente) y su calidad puede ser certificada, de acuerdo a normas internacionales, por una institución externa lo cual sería una garantía, tanto en el logro de la eficacia como de la eficiencia, efectividad y relevancia de la organización (Agudelo, 2012).

Debemos recordar que la evaluación autoreferencial no ha dado buenos resultados en nuestras instituciones educativas (Consorcio de universidades, 2005).

Debemos tener presente, también, que se certifica la calidad de los procesos, más no la calidad del producto. Al respecto, Nicoletti afirma "la estandarización de un sistema de calidad, no implica que los bienes, servicios o formación de los alumnos tengan que ser iguales en todo el mundo; la normalización de los sistemas y procesos apela al diseño particular de lo que la organización desea lograr, a la documentación de cómo se hacen las cosas y a la verificación de cómo se hicieron" (Nicoletti, 2008).

¿Cuál es el producto de la educación superior universitaria?, ¿̇cómo se puede certificar la calidad de ese producto?

\section{Finalidad de la educación}

De acuerdo a la Ley General de Educación 28044, son fines de la educación peruana formar personas para el logro de su realización y se integren a la sociedad, así como el desarrollo de sus capacidades y habilidades para vincular su vida con el mundo del trabajo. Además, contribuir a una sociedad forjadora de una cultura de paz considerando la diversidad de nuestro país, supere la pobreza, fomente la integración teniendo en cuenta los retos del mundo globalizado (MINEDU, 2003).

De lo anterior se puede apreciar que el enfoque de nuestra educación es formar personas con competencias laborales, capaces de contribuir al desarrollo de la sociedad peruana en un contexto globalizado.
La educación para cumplir con su finalidad debe ser de calidad. Pero la calidad es un proceso y como todo proceso se puede planificar, estructurar, enseñar, verificar y tomar acciones que nos lleven a mejorarla continuamente y a implantar una cultura de calidad (Presidencia del Consejo de Ministros, 2014).

\section{¿Educación con calidad para qué?}

El Comité de Calidad del SINEACE considera que la educación con calidad apunta al desarrollo de la condición humana en toda su complejidad, y por ende, al desarrollo del saber objetivo y del saber subjetivo, busca lograr que el saber objetivo devenga saber objetivado y el saber subjetivo saber recreado. Por calidad de la educación estamos entendiendo de manera global las distintas prácticas que apuntan a la socialización de las personas, a su inculturación, a todo lo que en su entorno refiere a su manera de entender la vida, a su cosmovisión, a sus subjetividades que van constituyendo en dichos procesos. No refiere únicamente al mundo de las instituciones educativas formales (SINEACE, 2015).

El SINEACE, más que concluir en una definición, delimita aspectos importantes que deben considerarse en una aproximación a la calidad educativa. Reconoce la educación con calidad como bien público al servicio de los ciudadanos, derecho humano fundamental que garantiza otros derechos y la centralidad del estudiante como sujeto de ese derecho (SINEACE, 2016).

Sin embargo, de acuerdo a la Ley 28740, la finalidad del SINEACE es garantizar a la sociedad que las instituciones educativas públicas y privadas, ofrezcan un servicio de calidad, con el propósito de optimizar los factores que inciden en los aprendizajes y en el desarrollo de las destrezas y competencias necesarias para alcanzar mejores niveles de calificación profesional y desarrollo (Congreso de la República del Perú, 2006).

Como se puede apreciar, la ley del SINEACE pone énfasis en alcanzar mejores niveles de calificación profesional (desempeño profesional) y desarrollo laboral (empleabilidad)

¿Cómo evaluamos si el profesional ha logrado las destrezas y competencias necesarias para alcanzar mejores niveles de calificación profesional y desarrollo laboral?

Es objetivo general de la Política de Aseguramiento, garantizar que todos los jóvenes del país tengan la oportunidad de acceder a un servicio educativo universitario con calidad, que ofrezca una formación integral y de perfeccionamiento contínuo, centrado en el logro de un desempeño profesional competente $y$, en la incorporación de valores ciudadanos que 
permitan una reflexión académica del país, a través de la investigación (26).

De acuerdo a este objetivo, lo central de un servicio educativo universitario con calidad es el logro de un desempeño profesional competente con valores ciudadanos... ¿̇ cómo evaluamos este logro?

¿Cómo se relaciona todo esto con un sistema de gestión de la calidad?

La Política de Aseguramiento incide más en la formación profesional con calidad tiene en el plan de gestión de la calidad la estrategia para la gestión de la calidad de los procesos de la formación integral, y en el plan de mejora continua los mecanismos para elevar la calidad de la formación académica. Mientras que el alcance del SINEACE va hacia excelencia, hacia el logro de un profesional con un desempeño de calidad lo cual lo vienen evaluando los Colegios profesionales mediante la certificación de competencias profesionales (27).

\section{Plan de gestión de la calidad como un indicador de las $\mathrm{CBC}$, y algo más}

Es necesario poner la debida atención a la trascendencia que tiene el plan de gestión de la calidad en las CBC; tal es así de importante, que ahora se exige que la universidad cuente con un área de gestión de la calidad y debe presentar la relación del personal calificado asignado a la misma (28).

Este indicador pone en relevancia, también, la importancia que tiene la gestión de la calidad bajo responsabilidad de personal calificado en este tema. Entonces żcuál es el marco referencial para diseñar un plan de gestión de la calidad?

Para poder diseñar un plan de calidad pertinente (con perspectiva y prospectiva) tenemos que tener muy presente a las cuestiones anteriores como, ¿̇qué es calidad y para qué?, żqué se entiende por educación con calidad o calidad educativa? de acuerdo con el marco normativo oficial vigente.

Pero también, es necesario tener presente que la verificación de las $\mathrm{CBC}$ tiene como fin conseguir el siguiente objetivo específico ...promover la eficacia, eficiencia e innovación en la educación superior universitaria.

Ferrás, concluye que la innovación "es un fenómeno cultural complejo que se traduce, desde el punto de vista de la gestión empresarial, en un concepto emergente de management, un concepto que integra conocimientos prexistentes (sobre estrategia, teoría organizativa, márketing, tecnología y liderazgo), y, a la vez que los integra, los supera a todos ellos en una nueva y superior visión de la realidad empresarial".
La innovación es un proceso sistémico, donde un cambio en una de las partes impacta y se valoriza en el conjunto, para diseñar arquitecturas organizativas que faciliten el trabajo transversal entre departamentos y articulando procesos internos de gestión de la innovación (Ferrás, 2014).

La capacidad innovadora emana fundamentalmente de las personas de la organización (cultura organizacional).

Se presenta una gran oportunidad para que la universidad peruana con calidad e innovación se internacionalize. Las tecnologías de la información y comunicación (TIC) son el medio apropiado. Algunas universidades peruanas ya están realizando los Massive Online Open Courses (MOOC, cursos online masivos y abiertos). La base es que la institución educativa sea una universidad con calidad acreditada y certificada.

Por lo tanto, el diseño de plan de gestión de la calidad debe tener en cuenta a la innovación para la gestión estratégica de la organización. Esto se puede lograr mediante el diseño (de acuerdo a la normatividad vigente) y la implantación de un SGC de acuerdo a alguna norma internacional.

Pero antes, es necesario conocer ¿̇qué es gestión de la calidad?

La gestión de la calidad implica un sistema y una administración de la calidad. El sistema de calidad se entiende como la estructura organizacional, los procedimientos, los procesos y los recursos necesarios para implantar la administración de la calidad. La gestión de la calidad forma parte de la función general de la dirección de la institución educativa que determina la política de calidad, los objetivos, las responsabilidades y la implantación de estos propósitos por medios tales como la planeación, el control, el aseguramiento y el mejoramiento de la calidad dentro del marco de ese sistema de calidad (Hernández, 1998).

Sistema de gestión de la calidad es aquella parte del sistema de gestión de la organización enfocada en el logro de resultados, en relación con los objetivos de la calidad, para satisfacer las necesidades, expectativas y requisitos de las partes interesadas, según corresponda (ICONTEC, 2009).

El Modelo de Licenciamiento define al plan de gestión de la calidad como documento que define una estrategia institucional orientada a la gestión de la calidad del servicio de educación superior universitario que brinda (SUNEDU, 2015).

Entonces esa estrategia del plan debe considerar la implementación de un SGC orientado no solamente 
al cumplimiento de la $\mathrm{CBC}$ sino también orientado a la estructura del Modelo de Aseguramiento cuya dimensión central es la formación integral (cuyos procesos son, la responsabilidad social, enseñanza y aprendizaje, y el de investigación) y, a su logro: el perfil del egreso.

¿Qué instrumentos se requiere para evaluar y certificar esa gestión de la calidad que se menciona en el plan?. Los componentes de las condiciones básicas de la calidad corren el riesgo de funcionar como áreas aisladas dentro de la organización sino se integran en procesos y se interrelacionan dentro de un sistema. La gestión de la calidad deber ser institucional, debe considerar a la institución como un sistema, sólo así adquiere significado y valor; por lo tanto el plan de gestión de la calidad debe considerar la implementación y certificación de un sistema de gestión institucional el cual se puede ir implementado en forma sistémica y sistemática empezando por los procesos claves y articuladores del programa como lo estamos presentando en la figura 1.

\section{Plan de gestión de la calidad e implementación de un SGC}

El licenciamiento y la acreditación son diferentes pero complementarios para el aseguramiento de la calidad. El licenciamiento es el control de la calidad mientras que la acreditación es garantía de calidad. El licenciamiento se refiere al cumplimiento de las condiciones básicas de calidad, mientras que la acreditación evalúa a la institución en función de sus propósitos declarados. El licenciamiento y la acreditación son esenciales para introducir en la universidad una gestión orientada a la calidad (SUNEDU, 2015).

Entonces un plan de gestión de la calidad debe ser transversal y contribuir no solamente al cumplimiento de las CBC sino también de la acreditación en su dinámica orientada hacia la excelencia académica. Un SGC debe asegurar la calidad en los procesos necesarios para que se cumpla la misión y los propósitos institucionales; es decir, la consistencia interna y la consistencia externa, la pertinencia de los propósitos y la capacidad de proponer, innovar y ampliar la oferta educativa (SUNEDU, 2015) en relación a sus referentes históricos institucionales, a su razón de ser (visión y misión) y a su factor diferenciador respecto al resto de instituciones educativas universitarias.

El Modelo de Acreditación tiene como un criterio el programa de estudios debe demostrar evidencia del funcionamiento del SGC en sus procesos principales y de las acciones para su evaluación y mejora. La estructura de este modelo tiene a la formación integral como una dimensión central; pero también considera al perfil del egreso como un eje central y articulador del programa de estudios. El perfil del egreso se identifica como parte de la gestión estratégica y conduce además la planificación del programa; orienta el proceso de formación integral; y el logro del mismo debe verificarse en cada egresado (SINEACE, 2016), ¿̇cómo?.

En consecuencia, la implementación de un SGC, de acuerdo a la norma ISO 9001, debe considerar al proceso de formación (que involucra la gestión estratégica y el soporte institucional) y, a la verificación del perfil del egreso como resultado de una formación integral con calidad.

La verificación del perfil del egreso como una dimensión de resultados es un proceso que diferencia al modelo actual del anterior. Esta dimensión tiene dos estándares: (SUNEDU, 2015). Logro de competencias, el objetivo es evaluar el cumplimiento de las competencias definidas en el perfil del egreso (es una condición para el egreso) (SINEACE, 2016). Seguimiento a egresados y objetivos educacionales, cuyo objetivo es el logro de los objetivos educacionales (SINEACE, 2016).

Si el cumplimiento del perfil del egreso es una condición vinculante para el egreso; entonces, el SGC debe considerar a este estándar como un proceso clave. Su logro reflejado en los índices de empleabilidad puede generar ventaja competitiva a la institución.

El programa de estudios debe establecer los mecanismos necesarios para el cumplimiento de estos estándares, definir sus procesos (evaluación del logro de competencias, monitoreo de la inserción laboral, desempeño profesional, satisfacción de empleadores y egresados, certificación de competencias, entre otros), evaluar la eficacia de esos procesos mediante el SGC y mejorarlos mediante planes de mejora. De ahí la importancia del plan de mejora.

De estos estándares podemos ver que la formación profesional se enlaza con el desempeño profesional (ejercicio profesional). Para garantizar la calidad de la formación profesional está la Política de Aseguramiento de la Calidad de la Educación Superior Universitaria (licenciamiento y acreditación) y, para evaluar la calidad del ejercicio profesional existe la evaluación y certificación de la competencias profesionales que vienen realizando los Colegios profesionales; sino, leamos lo que se dice en la definición de certificación de competencias profesionales.

La certificación es el reconocimiento público y temporal que se otorga a la persona que demuestra en su desempeño las competencias que ha adquirido luego de haber egresado luego de haber egresado del sistema educativo y haber trabajado un tiempo en esa área (Congreso de la República del Perú, 2006). 


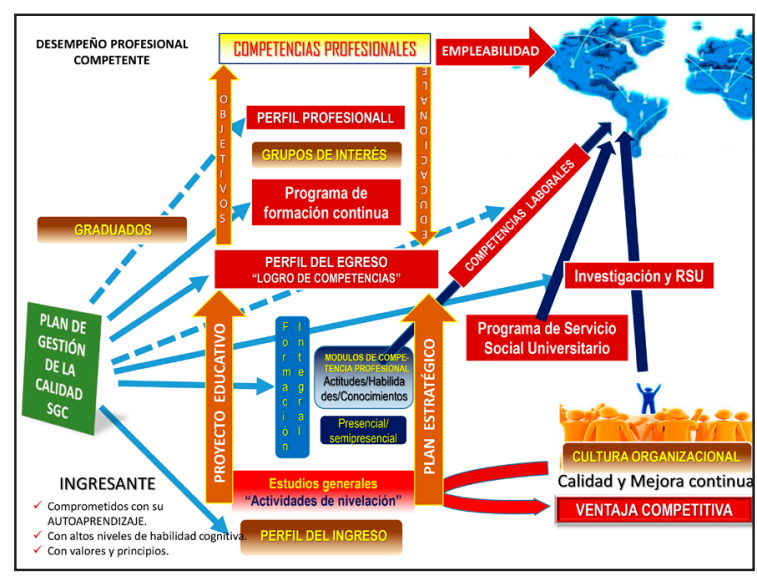

Figura 1. Actividades para el logro del objetivo institucional y nuestra propuesta de procesos clave que pueden ser certificados de acuerdo a la norma ISO 9001.

La figura 1 representa las actividades clave (procesos) para lograr una institución educativa con calidad acreditada y certificada.

También en la figura 1 se representa la importancia que tiene lograr el perfil del egreso con las capacidades para la empleabilidad. Entonces, es clave la determinación del perfil del egreso alineado a la demanda, en forma participativa de todos los actores de la comunidad universitaria, los grupos de interés, comité de expertos y particularmente los colegios profesionales porque los grupos de interés, para ambos, pueden ser los mismos.

De esta manera el aseguramiento de la calidad se extiende de la formación profesional al ejercicio profesional. El puente son los grupos de interés y el Programa de formación continua, que según la Ley Universitaria, es la encargada de actualizar los conocimientos profesionales, o desarrollar y actualizar determinadas habilidades y competencias de los egresados.

Además, la Ley Universitaria, en su artículo 109, indica que la universidad y los colegios profesionales deben mantener una actitud vigilante en cuanto a la calidad del ejercicio profesional de sus afiliados, y deben establecer mecanismos orientados a supervisar y promover el ejercicio eficiente de la profesión.

Por tanto, el sistema de aseguramiento de la calidad de la educación superior universitaria se articula con los Colegios profesionales.

\section{Estrategias para la implementación de un SGC para los procesos y el producto}

Teniendo en cuenta todo lo mencionado hasta aquí, ya podemos diseñar una estrategia para la implementación de un SGC pertinente con el nuevo contexto educativo de licenciamiento y acreditación. El propósito de esta publicación no es dar instrucciones ni repetir la metodología de cómo se implementa un SGC; sino, en base a nuestra experiencia en la docencia y de haber implementado SGC, de acuerdo a la norma ISO 9001, en varias instituciones educativas, podemos dar algunas pautas para asegurar el éxito de su implantación y desarrollo sostenible.

La implantación de un SGC, no debe ser por moda, obligación, status, marketing; debe ser para demostrar con evidencias que las actividades de la organización se hacen de manera congruente y con mejora continua para lograr la excelencia en la gestión de la profesionalización del talento humano para la empleabilidad, la generación de conocimiento con calidad y el desarrollo de la innovación en nuestro país.

La implantación de un SGC en un programa de estudios de educación superior universitario debería tener como objetivo principal certificar el proceso de formación integral, de acuerdo a SINEACE, como una dimensión central para lograr el perfil de egreso. El proceso de formación incluye la responsabilidad social, el proceso de enseñanza y aprendizaje y el de investigación. El perfil de egreso se convierte en un eje central y articulador del programa de estudios (SINEACE, 2016).

Toda organización es un sistema vivo y su gestión es el resultado de las interacciones de sus personas, de la eficacia de sus procesos y con el tiempo van desarrollando un sistema de calidad y, una cultura. El sistema de calidad puede ser diagnosticado, evaluado, mejorado y certificado; es decir, gestionado.

Entonces, antes de implantar un SGC de acuerdo a la norma ISO 9001 se requiere hacer un diagnóstico de la calidad del sistema de gestión de la organización y para el diseño del SGC se requiere tener en cuenta el marco normativo actual que el Estado ha determinado para el aseguramiento de la calidad de la educación superior universitaria en el Perú.

Entonces, implantar un SGC en una institución de educación superior universitaria adquiere una connotación particular que lo diferencia de las demás organizaciones. 


\section{Importancia y metodología del diagnóstico de la calidad del sistema de gestión}

El diagnóstico muestra el análisis del estado actual de diseño e implementación del sistema de gestión existente de la universidad en comparación con los requisitos de la norma internacional ISO 9001, la misma que brinda los requisitos para la implementación de un SGC.

Asimismo, el diagnóstico cumple los siguientes objetivos:

- Conocer la situación actual de la universidad con el fin de evaluar sus fortalezas y debilidades en materia de gestión de calidad con respecto a los requisitos establecidos por la Norma ISO 9001 identificando brechas por cada requisito.

- Orientar a la universidad acerca de la preparación y acciones necesarias para alcanzar la conformidad del SGC a ser implementado respecto a los estándares referidos.

- Elaborar el Plan de Trabajo (Gantt) detallado y específicamente orientado a las necesidades de la universidad para la implementación de su SGC.

Para ello se realiza entrevistas a distintos niveles jerárquicos de la universidad, así como la revisión de documentos y registros de acuerdo a un programa de diagnóstico elaborado por el consultor líder el cual debe ser aprobado por la universidad previamente antes de la ejecución del servicio.

Todo ello con el objeto de obtener mediante un muestreo información acerca de las actividades que realiza la organización, su infraestructura, el ambiente de trabajo, procedimientos y prácticas de trabajo. Así como, conocimiento sobre el sistema interno de información y la existencia de sus documentos y registros.

Por lo general, el diagnóstico tiene una duración entre 3 a 5 días para llevarlo a cabo en las instalaciones de la universidad.

Como dijimos al principio, el nuevo marco normativo que ha marcado un antes y un después en el contexto de la educación superior universitaria en nuestro país. Este nuevo marco normativo hace que institución de educación superior universitaria adquiere una connotación propia que lo hace diferente de las demás organizaciones.

Por eso no es lo mismo implementar un SGC en una universidad que en una empresa de manufactura.

Ignorar la importancia que tiene el diagnóstico es un error frecuente que vienen cometiendo las instituciones universitarias en la implantación de un SGC en base a la norma ISO 9001.

Varias instituciones educativas ya tienen el SGC, implementado y certificado de acuerdo a la norma ISO 9001:2008, ahora, tendrán que hacer cambios, no solamente por la nueva versión del ISO 9001:2015, sino porque los procesos se tienen que adaptar a las nuevas condiciones básicas de calidad y deben estar orientadas para alcanzar los nuevos estándares del Modelo de Acreditación.

Nuestra propuesta es que el diagnóstico, también, debe alcanzar a la descripción de la cultura organizacional.

\section{Cultura organizacional}

Fischman, nos dice "toda empresa, quiéranlo o no, ya tienen una cultura formada. Esta cultura puede ayudarlas a competir o perjudicarlas en la competencia. Cambiar la cultura es un reto muy difícil, sobre todo para aquellas organizaciones que ya la tienen varios años" (Fischman, 2009). Para Schein,"la cultura es el conjunto de supuestos y creencias aprendidas sobre la cual las personas basan sus conductas diarias" (Schein, 1999).

Las organizaciones de éxito le dan un gran valor a su cultura organizacional, tal es así que han creado el puesto de "guardianes de la cultura organizacional".

Según Fishman, existen quienes piensan que la cultura ideal es la estrategia que la empresa necesita. La cultura debe servir para que la empresa obtenga las ventajas competitivas que necesita (figura 1). Pero además, cree que ciertos rasgos culturales o supuestos funcionales comunes en empresas exitosas, dicho de otro modo existen supuestos disfuncionales que si se encuentran en la cultura van a perjudicar la estrategia de la empresa (Fischman, 2009).

Entonces, el diagnóstico de una organización debe determinar los supuestos culturales compartidos y esos supuestos disfuncionales que pueden limitar el desarrollo de una cultura de calidad en la organización educativa.

Para facilitar esta aproximación, primeramente, hay que definir żcuál es la naturaleza de la organización educativa y cuál es la conceptualización del liderazgo?

\section{La organización educativa como un sistema complejo y liderazgo}

Morin define a la organización como la disposición de relaciones entre componentes o individuos que produce una unidad compleja o sistema, dotado de cualidades desconocidas en el nivel de los 
componentes o individuos. La organización une de forma interrelacional elementos 0 eventos o individuos diversos que a partir de ahí se convierten en componentes de un todo. Asegura solidaridad y solidez relativa a estas uniones, asegura pues, al sistema una cierta posibilidad de duración a pesar de las perturbaciones aleatorias. La organización, pues: transforma, reúne, mantiene (Morin, 1993).

Si la organización es un sistema complejo, entonces cuáles son las implicaciones para la conceptualización del liderazgo, tan necesario en la transformación que se requiere de las instituciones educativas en nuestro país.

Contreras y otros (2012), refuerzan la definición de la organización como un sistema adaptativo complejo, que tiene un gran número de agentes interdependientes que se encuentran en permanente interacción dinámica entre sí y con el entorno, y que a través de procesos de autoorganización y acumulación de experiencia lograr crear propiedades emergentes que les permiten adaptarse y sobrevivir a las condiciones cambiantes del ambiente o al surgimiento de nuevas pautas. En este sentido, el liderazgo (fenómeno complejo) en las organizaciones (sistemas complejos) es abordado desde una perspectiva más integral e incluyente en cuanto permite considerar todos los elementos involucrados en el proceso de liderazgo, que favorecen la adaptación del sistema, la innovación y el aprendizaje.

\section{Cultura organizacional y cultura de la calidad}

Para la directora del Centro Interuniversitario de Desarrollo (CINDA), María José Lemaitre, "La principal preocupación de una institución no debería ser la acreditación, sino mejorar su calidad (...) Lo importante es que pasar por este proceso, represente el compromiso real y permanente por mejorar. Ello se traduce en "generar una cultura de calidad" (Lemaitre, 2013).

La resistencia al cambio es elevado en nuestras organizaciones educativas. La cultura de calidad es un conjunto de valores y procesos que se orientan al cumplimiento de la misión y propósitos de la organización de acuerdo a las normas establecidas y al entorno socio cultural.

Olea, afirma que algunos factores que resultan importantes en el cambio de cultura hacia la calidad son: a) crear conciencia en los trabajadores sobre los principios de la calidad; b) obtener el compromiso de todos los miembros de la organización; c) lograr que los miembros de la organización se identifique con el sistema de gestión; y d) educación y entrenamiento en sistemas de gestión de la calidad (Olea, 1995).
Para Vesga, el análisis y comprensión de la cultura organizacional se hace necesario como punto de partida para la implementación de sistemas de gestión de la calidad (Vesga, 2011).

Serna, afirma que "uno de los mayores errores gerenciales está en separar la cultura organizacional de las estrategias corporativas. Es importante que, cuando estas se definan, incluyan programas y acciones que coincidan $o$, si se requiere, modifiquen los elementos culturales que impidan o faciliten la puesta en marcha de las estrategias" (Serna, 2008).

Nosotros coincidimos con estos autores y creemos que una estrategia para implementar el cambio en una organización hacia una cultura de calidad comprende prioritariamente la gestión del capital humano mediante la gestión de la cultura organizacional (figura 2).

El Modelo de Licenciamiento tiene como un componente de la Condición I a los Sistemas de información; así

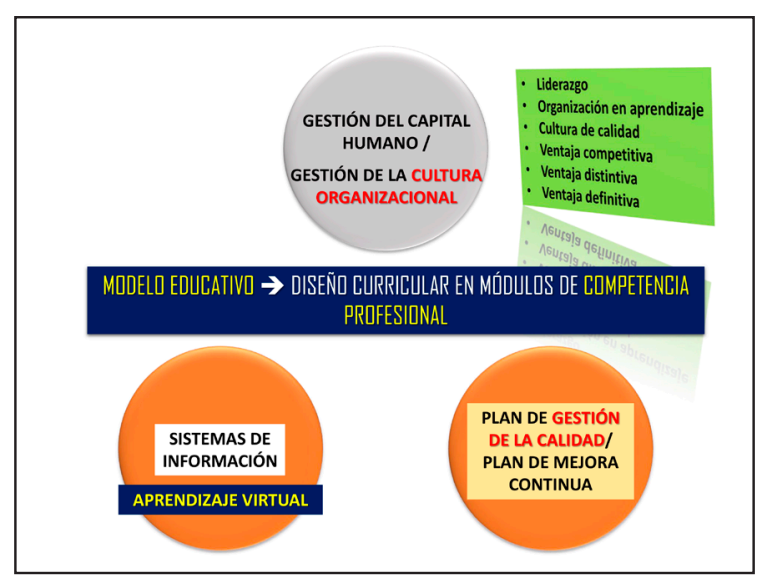

Figura 2. Sistemas básicos para iniciar el cambio en una organización universitaria: sistema de información, SGC, y la gestión de la cultura organizacional.

como al Plan de Gestión de la Calidad institucional. El Modelo de Acreditación, considera dos estándares referidos al aseguramiento de la calidad, sistema de gestión de la calidad (SGC) y planes de mejora; en lo referente a los recursos humanos indica que el programa de estudios gestiona los recursos humanos de acuerdo con los perfiles de puestos y funciones establecidas.

Sin embargo, como ya hemos visto, la cultura organizacional es algo más.

Nuestra propuesta es que para iniciar el cambio en una organización educativa se debe tener en cuenta tres sistemas: el sistema de información, el SGC y la gestión de la cultura organizacional (figura 2). 


\section{Reflexiones finales de nuestra propuesta}

La dirección estratégica de las organizaciones educativas tienen que hacer uso de todas las herramientas de gestión para lograr la profesionalización del talento humano para la empleabilidad, la generación de conocimiento con calidad y el desarrollo de la innovación en nuestro país. El plan de gestión de la calidad y el plan de mejora continua se convierten en herramientas estratégicas para el cambio de una organización funcional hacia una sistémica basada en procesos.

En el nuevo marco del aseguramiento de la calidad de la educación superior universitaria, la implantación de un SGC de acuerdo a la norma internacional ISO 9001 , debe ser a los procesos y a los resultados. Por eso nosotros proponemos, inicialmente, la implementación y certificación de cuatro (macro)procesos: 1) perfil del ingreso, 2) formación integral, 3) perfil del egreso, 4) programa de educación continua. Adicionalmente, se puede implementar el proceso para la formación por competencias laborales si la organización opta por esta modalidad (figura 1).

El diagnóstico de la calidad del sistema de gestión es imprescindible toda vez que muestra el análisis del estado actual de diseño e implementación del sistema de gestión existente de la universidad en comparación con los requisitos de la norma internacional ISO 9001, la misma que brinda los requisitos para la implementación de un SGC. El diagnóstico, también, debe alcanzar a la caracterización de la cultura organizacional necesaria para la gestión del factor humano y la generación de la ventaja competitiva de la organización.

El diagnóstico de una organización debe determinar los supuestos culturales compartidos y esos supuestos disfuncionales que pueden limitar el desarrollo de una cultura de calidad en la organización educativa. Creemos que la estrategia para desarrollar una cultura de calidad debe tener en cuenta prioritariamente la gestión del capital humano; por esa razón proponemos tres sistemas clave de inicio para el cambio organizacional: el sistema de información, el SGC y la gestión de la cultura organizacional (figura 2).

\section{CONCLUSIONES}

De todo lo anterior se concluye que la implementación de un sistema de gestión de la calidad en una universidad y sus programas de estudio requiere de una estrategia diferente, toda vez que las organizaciones educativas tiene su propia normatividad y contexto, con grupos de interés que hablan el mismo lenguaje. Por lo tanto, esta implementación requiere de profesionales que tengan conocimiento certificado del modelo de licenciamiento, la política de aseguramiento de la calidad de la educación superior universitaria, del nuevo modelo de acreditación de institucional para universidades y del modelo de acreditación para programas de estudio de educación superior universitaria. No tener en cuenta estas consideraciones puede ocasionar un fracaso del proyecto y la pérdida de recursos valiosos para la organización educativa.

\section{REFERENCIAS BIBLIOGRÁFICAS}

Agudelo Tobón, L. F. (2012). Evolución de la Gestión por Procesos. Instituto Colombiano de Normas Técnicas y Certificación, ICONTEC.

Benzaquén de Las casas, J. B. (2013). Calidad en las empresas latinoamericanas: El caso peruano. Globalización, Competitividad y Gobernabilidad. 7(1), 41-59.

Congreso de la República del Perú. (2006). Ley del Sistema Nacional de Evaluación, Acreditación y Certificación de la Calidad Educativa. Lima, Perú: Congreso de la República.

Consejo de Evaluación, A. y. (2008). Modelo de Calidad para la Acreditación de Carreras Profesionales. 12, 20. Perú: CONEAU.

Consejo de Evaluación, Acreditación y Certificación de la Calidad de la Educación Superior Universitaria - CONEAU. (2012). Criterios y estándares para la certificación profesional en el Perú. Obtenido de https://www.sineace.gob.pe/wp-content/ uploads/2015/01/04-Compendio-TecnicoNormativo-2012.pdf

Consorcio de universidades. (2005). Gestión de la calidad para instituciones de educación superior. Procesos de autoevaluación y acreditación. (147), Primera edición.

Contreras, T. F., Barbosa , R. D., \& Castro , R. G. (2012). La organización como sistema complejo: implicaciones para la conceptualización del liderazgo. Criterio Libre, 10 (16), 193-206.

Evoluciona . (Noviembre de 2015). No es la más fuerte de las especies la que sobrevive.

Ferrás, X. (2014). Las seis olas de la innovación, żen cuál opera usted? (14), 16.

Fischman, D. (2009). Cuando el liderazgo no es suficiente. Desarrolle su inteligencia cultural en la empresa. Universidad Peruana de Ciencias Aplicadas (UPC).

Hernández Ruiz, R. (1998). ¿̇Certificación ISO 9000 en Educación?. Colección Pedagógica Universitaria (Vol. 30). Obtenido de http://www.uv.mx/cpue/ colped/N 30/Certificaci\%C3\%B3n.htm

Instituto Colombiano de Normas Técnicas y Certificación - ICONTEC . (2009). Normas fundamentales sobre gestión de la calidad y documentos de orientación para su aplicación. Colombia, Bogotá : ICONTEC.

Lemaitre, M. (2013). "Hay que generar una cultura de calidad". Obtenido de http:// 
noticias.universia.edu.pe/vida-universitaria/ noticia/2013/05/28/1026189/maria-joselemaitre-hay-generar-cultura-calidad.html

Ministerio de Educación - MINEDU . (2003). Ley General de Educación. Lima, Perú: MINEDU.

Ministerio de Educación - MINEDU. (26 de setiembre de 2015). Política de aseguramiento de la calidad de la educación superior universitaria. El Peruano, pág. 562360.

Morin, E. (1993). El Método. I. La naturaleza de la naturaleza. Madrid: Cátedra.

Morin, E. (2002). Los siete saberes necesarios para la educación del futuro. Buenos Aires: : Nueva Visión.

Nicoletti, J. A. (2008). Adecuación y aplicación de las normas de calidad ISO 9000:2000 en el campo educativo. 3 , 2, 79. Horizontes educacionales.

Olea, B. (1995). Dirigir el cambio de la cultura organizacional hacia la calidad total. 28, 11-18. Cuadernos.

Presidencia del Consejo de Ministros - PCM. (2013). Política Nacional de Modernización de la Gestión Pública al 2021 . 26, 32. Lima, Perú: PCM.

Presidencia del Consejo de Ministros - PCM. (2014). Política Nacional para la Calidad. El Peruano, págs. 566593, 566594.

Schein, H. (1999). Corporate Culture: Survival Guide. 24. San Francisco: Jossey-Bass.

SEMANAeconómica.com. (Julio de 2014). Mejorar la eficiencia es la principal prioridad de los gerentes generales. Obtenido de http://semanaeconomica. com/article/management/gerencia/164892 desaceleracion-economica-en-el-peru-como-lacombaten-las-empresas/

Serna, H. (2008). Gerencia estratégica. Bogotá D.C: 3R Editores.

Sistema Nacional de Evaluación, Acreditación y Certificación de la Calidad Educativa - SINEACE. (2015). Calidad en educación y derroteros. 29, 30. Lima, Perú: SINEACE.

Sistema Nacional de Evaluación, Acreditación y Certificación de la Calidad Educativa - SINEACE. (2016). Modelo de Acreditación para Programas de Estudios de Educación Superior Universitaria. 25, 18. Lima, Perú: SINEACE.

Superintendencia Nacional de Educación Superior Universitaria - SUNEDU. (2015). El modelo de licenciamiento y su implementación en el Sistema Universitario Peruano. 10-17. Lima, Perú: SUNEDU.

Universidad de Ingeniería y Tecnología - UTEC. (Marzo de 2016). UTEC es la primera universidad en obtener Licencia Institucional de la SUNEDU. Obtenido de https://www.utec.edu.pe/noticias/ utec-es-la-primera-universidad-en-obtenerlicencia-institucional-de-la-sunedu

Vesga, J. J. (2011). Memorias del Seminario Gestión de la Cultura Organizacional. Cali: Editorial Bonaventuriana. 